\title{
A highly integrated MMIC Chipset for 40 GHz MVDS Applications
}

\author{
O. Vaudescal, D. Kabat, A.M. Couturier, R. Sevin, C. Dourlens, P. Quentin \\ United Monolithic Semiconductors ( UMS ) \\ Route Départementale 128, BP 46, 91401 ORSAY Cedex, France \\ Tel. 33-1-6933-0582 / Fax. 33-1-6933-0552 / e.mail olivier.vaudescal@ ums-gaas.com
}

\begin{abstract}
This paper is intended to give an insight in the development of PHEMT multifunction MMICs (MFC) in the millimetre wave frequency range. It applies to the fast growing Multipoint Video Distribution System (MVDS) market, offering a five-chip solution for the subscriber transceivers. The focus is on the local oscillator, the up-converter and the down converters at 40GHz. These two last chips include a balanced mixer, a buffer amplifier for the local oscillator and a low noise amplifier used either as a LNA (for Rx) or as a transmit driver (for Tx). The design was oriented toward low chip-size in order to reach the cost requirement for such systems. Results are shown from a demonstrator assembly built with these five MMICs.
\end{abstract}

\section{INTRODUCTION}

The availability of millimetre-wave short haul communication systems opens the door to a low-cost and truly broadband wireless access to interactive multimedia services (telephone, video, computer data), targeting residential family entertainment as well as business operations. In western Europe, the so-called MVDS system (Multipoint Video distribution System) is actively developed for this purpose, and will be operated in the 40.5-42.5 GHz or 40.5-43.5 GHz frequency band.

In order to address the consumer market, extremely low prices for the millimetre-wave front-end of the subscriber terminals are requested, and, in our view, this will be feasible only with a highly integrated and full MMIC solution, which will drastically simplify the assembly of the front-end module and reduce its cost.

In this paper, we present the performances of a $42 \mathrm{GHz}$ MMIC chipset developed for a MVDS transceiver module. With this chipset, the millimetrewave front-end of a transceiver module can be easily built, using only the five MMICs of this chipset; it is described in the next section.

\section{CHIP-SET DESCRIPTION}

\section{A. Description of the $40 G H z$ MVDS MMIC chipset}

The block diagram of the MVDS transceiver frontend is given in figure 1.

It is composed of a local oscillator, a receiver and a transmitter (for the return path). This transceiver has been designed to use the MVDS $40.5-42.5 \mathrm{GHz}$ frequency band and to be fully compatible with the DTH (Direct-To-Home) standard format for the satellite digital TV broadcast, using the same $0.95-2.15 \mathrm{GHz}$ intermediate frequency (IF) band.

A full MMIC solution has been retained, and four complex multifunction MMICs have been designed:

- a local oscillator MMIC, including a $10 \mathrm{GHz}$ DRO and two cascaded multipliers;

- a receiver MMIC, including a LNA and a downconverter mixer ;

- an transmitter MMIC, including an up-converter mixer and a driver amplifier ;

- an amplifier (DRIV40) for power transmission.

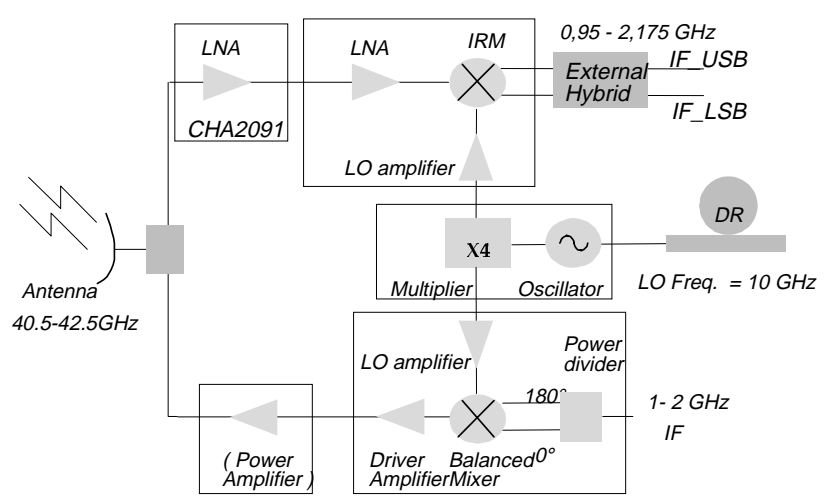

Fig.1: Block diagram of the transceiver of the MVDS subscriber terminal 
All the four MMICs and the CHA2091 (used as the first stage in the receiver path) use UMS pseudomorphic HEMT MMIC process (PH25) with $0.25 \mu \mathrm{m}$ Al gates, dedicated to very low-noise and small/medium power circuits up to $40 \mathrm{GHz}$. The typical noise figure of $\mathrm{PH} 25$ transistors is $1.5 \mathrm{~dB}$ with $8 \mathrm{~dB}$ of associated gain at $40 \mathrm{GHz}$.

The photograph of the demonstrator assembly is given in figure 2 . The purpose of this assembly is to demonstrate the functionality of the MMICs and not at all to make a real radio for MVDS. The local oscillator (LO) is placed in the left cavity with an external resonator while the RF input (top) and RF output (bottom) are available on the right hand. For the receiver, we use an external SMD broadband quadrature coupler.

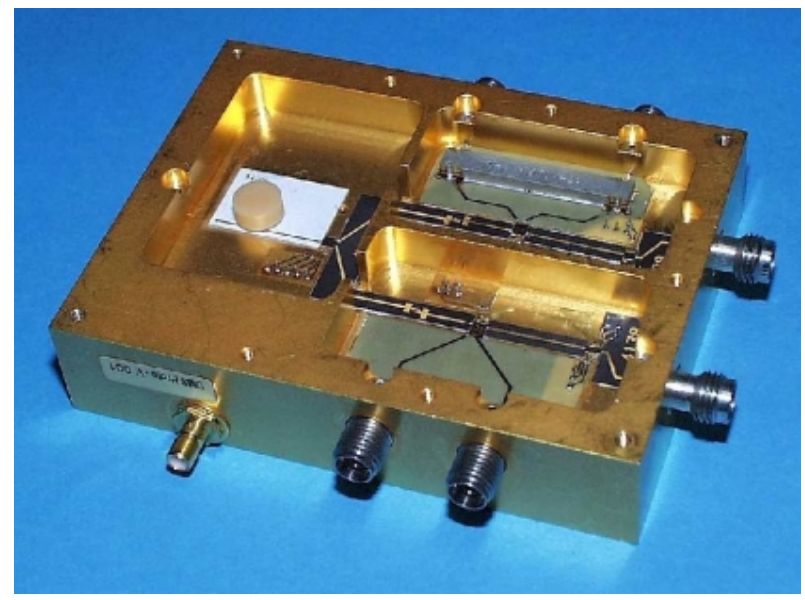

Fig.2: Photograph of the MVDS transceiver demonstrator at $40 \mathrm{GHz}$.

\section{B. Local oscillator MMIC}

This MMIC integrates an X-band DRO and a cascade of two frequency multipliers by 2 as described by the schematic of the figure 3 .

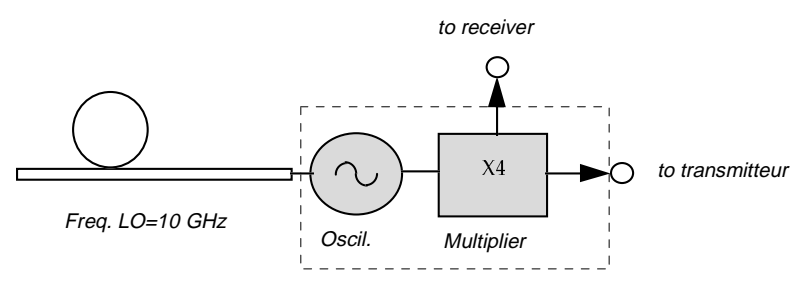

Fig.3: Principle of the local oscillator

The layout of this oscillator is presented in figure 4 .

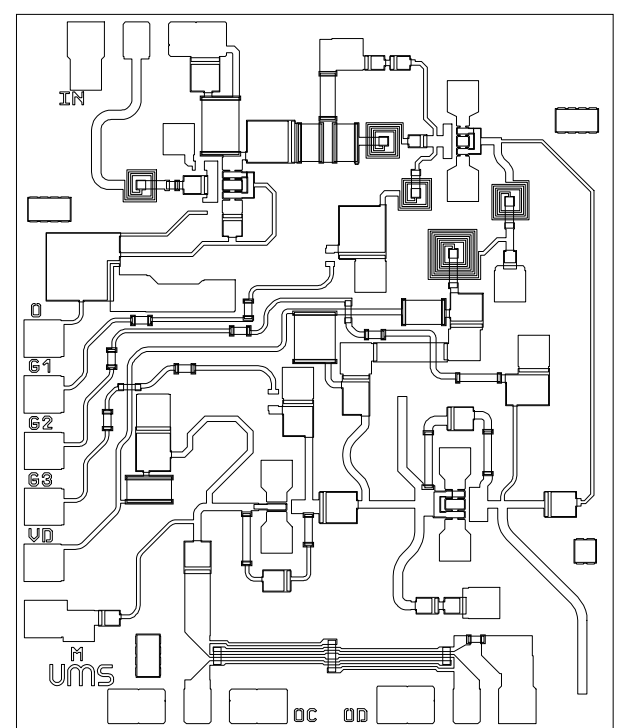

Fig.4 : Layout of the local oscillator. The chip size is $3.4 \mathrm{~mm}^{2}$

The oscillator circuit is stabilised by an off-chip dielectric resonator. It can be adjusted from $9.75 \mathrm{GHz}$ to $10.25 \mathrm{GHz}$. After frequency multiplication, this circuit provides $11 \mathrm{dBm}$ of power in the LO frequency band 39.5-40.5GHz.

The rejection at the first sub-harmonic at $20 \mathrm{GHz}$ is higher than $30 \mathrm{dBc}$ and the rejection of the second harmonic is higher than $16 \mathrm{dBc}$.

The output signal is split in two ways in order to feed the up- and down-converter circuits by an on-chip Lange coupler providing $+8 \mathrm{dBm}$ for each port. The phase noise measured at $100 \mathrm{kHz}$ from carrier at $39.5 \mathrm{GHz}$ is $100 \mathrm{dBc} / \mathrm{Hz}$.

\section{Receiver circuits}

The receiver path is composed by two MMIC circuits.

The first one is a MFC down-converter which integrates a $40 \mathrm{GHz}$ LNA, a $40 \mathrm{GHz}$ local oscillator buffer amplifier and an image rejection mixer (IRM). The schematic is resumed by the figure 5 .

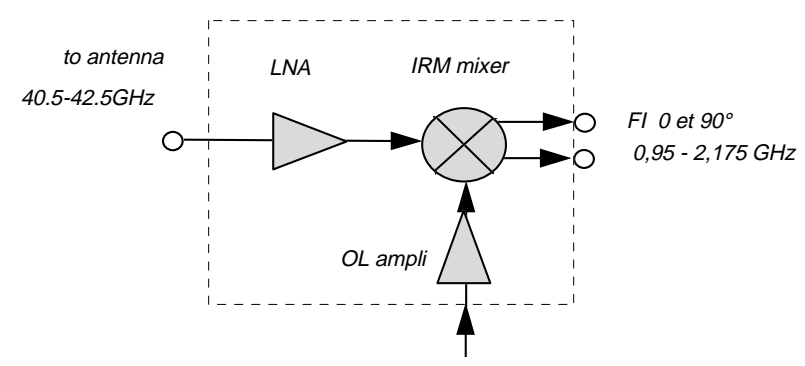

Fig.5 : Principle of the receiver down-converter 
The mixer is based on cold FETs structures. The benefit is to not need any drain voltage supply and so to simplify the DC supply design.

To realise a SSB mixer, only two individual cells balanced in quadrature are requested.

The in-phase and $90^{\circ} \mathrm{RF}$ signals are obtained using a Lange coupler and the IF signals must be recombined off-chip for image frequency rejection. The layout of this MMIC is displayed in figure 6.

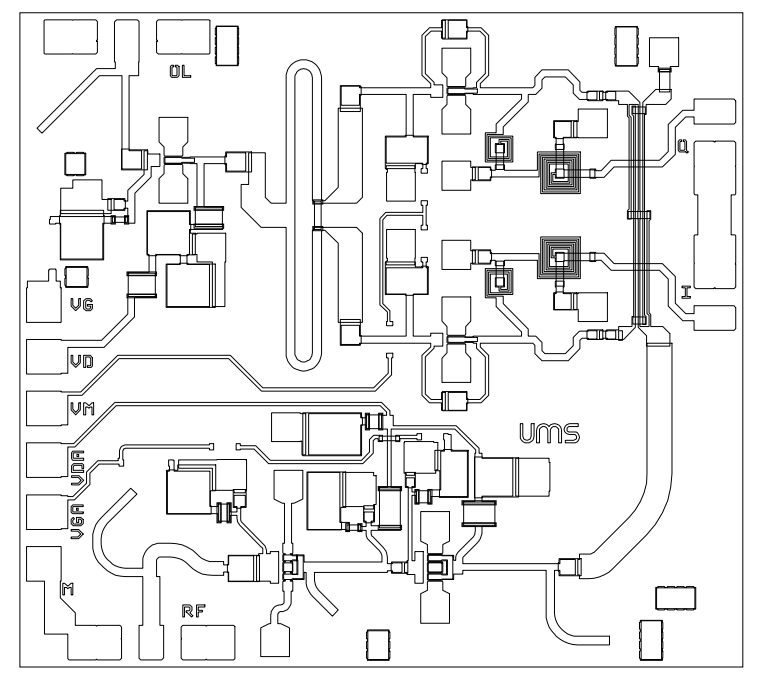

Fig.6 : Layout of the down-converter MFC. The chip size is $4.3 \mathrm{~mm}^{2}$

The second MMIC circuit is the UMS catalogue product low noise amplifier CHA2091 (figure 7).

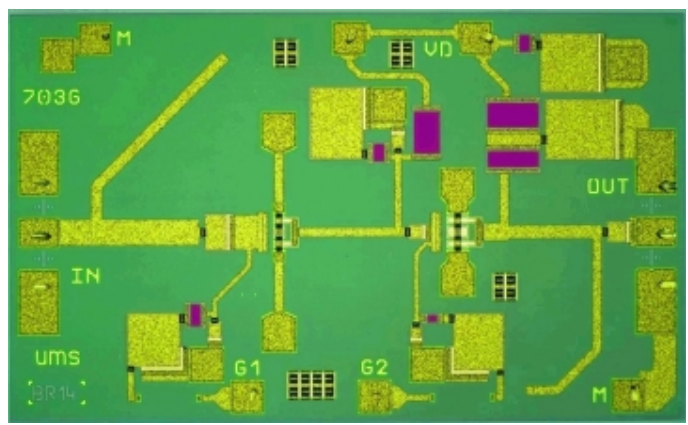

Fig.7: Photograph of the LNA CHA2091

This two-stage amplifier exhibits a $14 \mathrm{~dB}$ gain and a $3.0 \mathrm{~dB}$ noise figure in the 36 to $40 \mathrm{GHz}$ frequency band. The use of this amplifier can be extended up to $43 \mathrm{GHz}$, and so be used for these applications.

The overall measured receiver path conversion gain is higher than $20 \mathrm{~dB}$ in Upper Side Band (USB), and the image frequency rejection is better than $20 \mathrm{dBc}$. The results in Lower Side Band (LSB) are almost the same but the slope is different due to a different combination of the frequency slope of the IF filter, of the RF one and of the CHA2091 itself. The overall Noise Figure is lower than $4.0 \mathrm{~dB}$, accounting for a $1.0 \mathrm{~dB}$ degradation brought back by the MFC down-converter (compared to the $3.0 \mathrm{~dB}$ of the CHA2091 itself).

The LO buffer amplifier of the MFC is useful to isolate the mixer from the external (assembly) and also to reduce the required power for the LO (typically $0 \mathrm{dBm})$ that may radiate from the bonding wire. This feature is very important to reduce the level of the spurious in the radio, as the specifications are more and more demanding on these aspects.

Two accesses are provided for the IF in order to use the IF external quadrature combiner for the image suppression.

The conversion gain and image rejection results shown in figure 8 include all the effects of the assembly and external hybrid combiner (shown in figure 2).

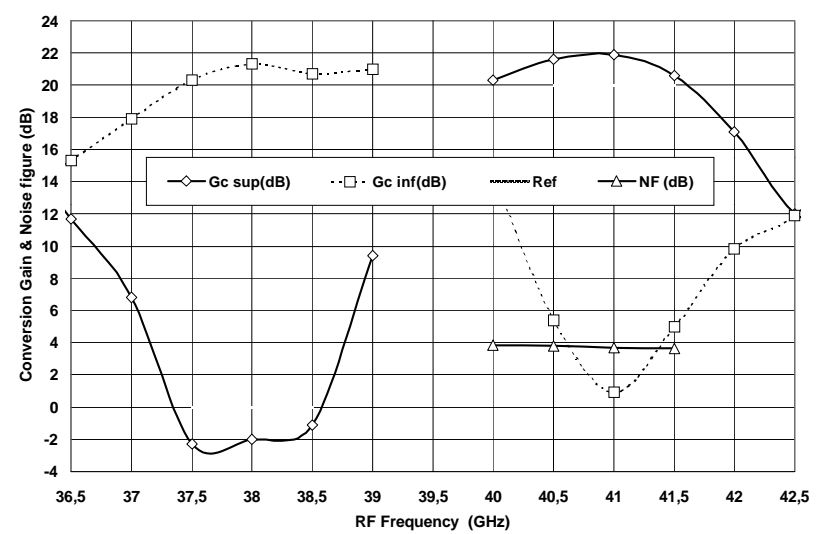

Fig.8: Full Hybrid measurement: Conversion Gain (USB \& LSB), Image Rejection and Noise Figure

\section{Transmitter circuits}

The transmitter path is composed also by two MMIC circuits.

The first one is a MFC up-converter which integrates a LO buffer amplifier, an IF input splitter circuit, a balanced mixer for LO rejection, and a two-stage driver amplifier. These four parts are described by the schematic of the figure 9 below.

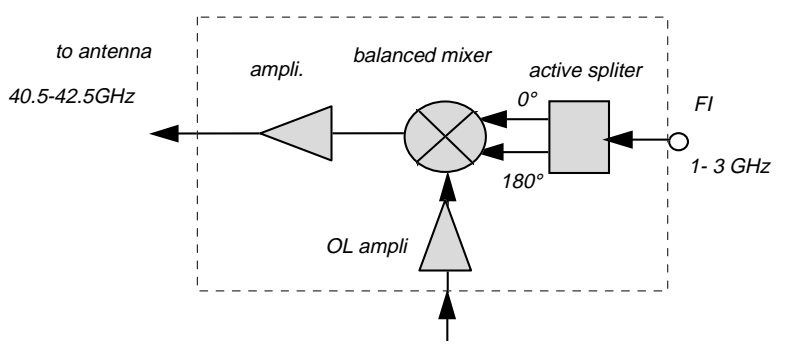

Fig.9: Principle of the receiver up-converter 
The layout of this MMIC is displayed below in figure 10.

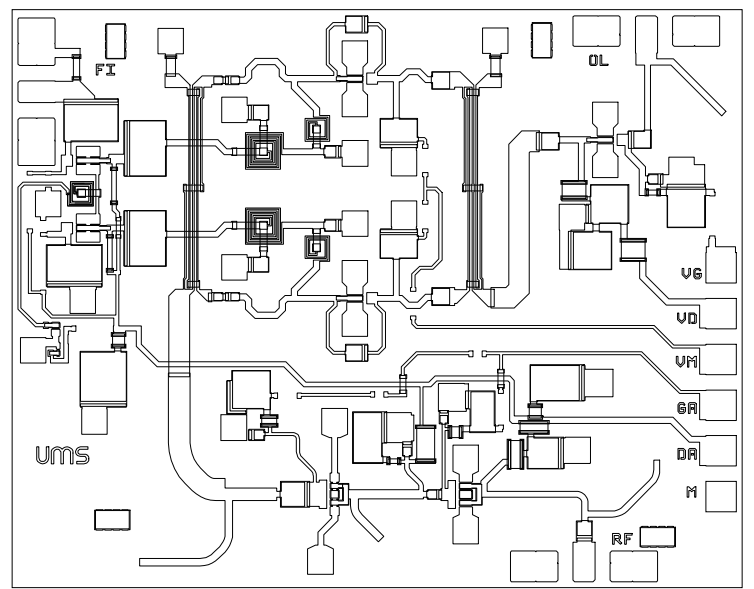

Fig.10: Layout of the up-converter MFC. The chip size is $4.9 \mathrm{~mm}^{2}$

The balanced mixer is composed of two cold FETs biased at pinch-off and placed between two Lange couplers to split the LO signal, recombine the RF signal and reject the LO signal on the output port. The input splitter circuit provides the mixer with the in phase and inverted IF signals.

The second MMIC circuit is the medium power amplifier described by the photograph of the figure 11 . It provides the transmitted power of the return path signal. It is a three-stage amplifier: the gain is higher than $16 \mathrm{~dB}$ with an output $1 \mathrm{~dB}$ compression point about $16-17 \mathrm{dBm}$.

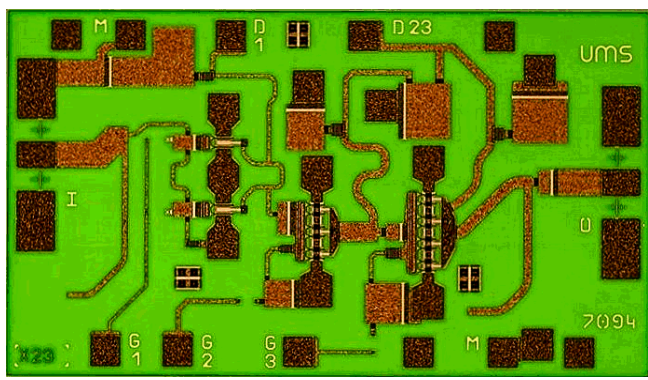

\section{DRIV40}

Fig.11: Photograph of the transmitter medium power amplifier

The overall conversion gain is better than $20 \mathrm{~dB}$ up to $3 \mathrm{GHz}$ IF and the $\mathrm{LO}$ to $\mathrm{RF}$ isolation at the RF port is better than $20 \mathrm{dBc}$ for $-20.0 \mathrm{dBm}$ IF input power. The Pout for $1 \mathrm{~dB}$ gain compression is achieved for an IF input power of $-6 \mathrm{dBm}$ at an RF frequency equal to $43 \mathrm{GHz}$. The figures 12 and 13 show the behaviour of this circuit respectively in the frequency band and for the non-linear performances.

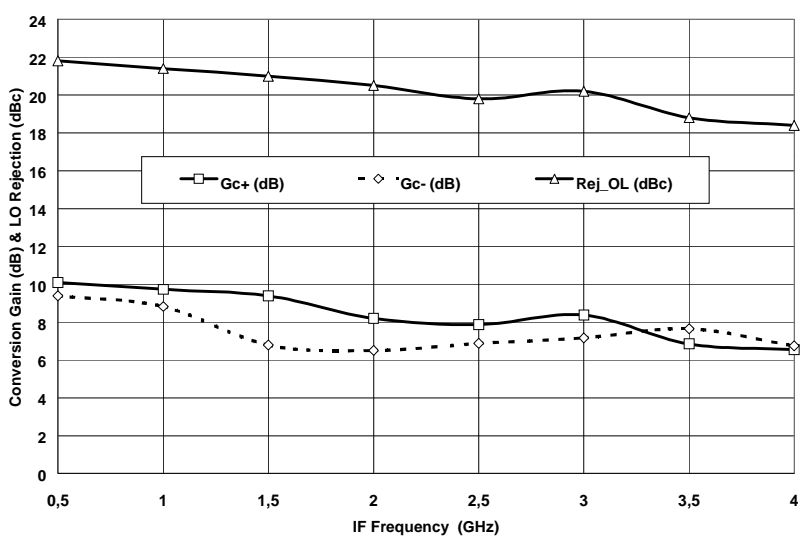

Fig.12: Full Hybrid Measurement: Up-Converter conversion gain and LO suppression

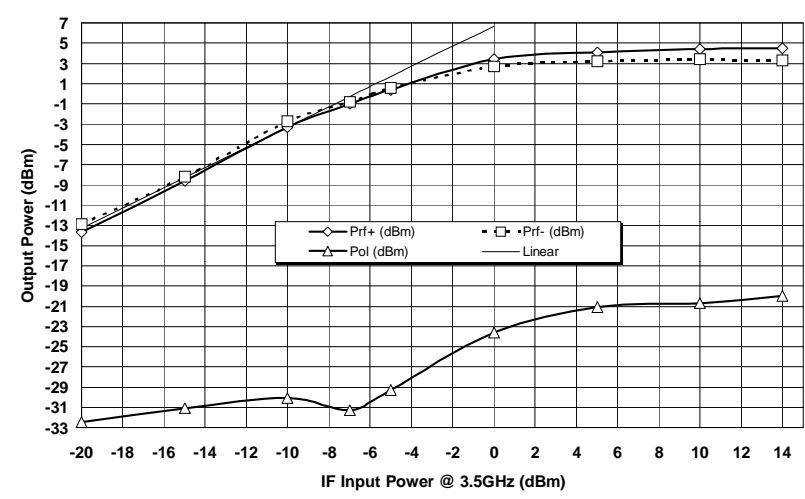

Fig.13: Full Hybrid Measurement: Up-Converter Pin/Pout and LO leakage @ 43 GHz RF

\section{CONCLUSION}

We have successfully demonstrated the development of a chipset including three complex $40 \mathrm{GHz}$ multifunction MMICs, providing a full MMIC solution for the fabrication of a transceiver module of a MVDS subscriber terminal.

\section{ACKNOWLEDGEMENTS}

The development of the MMIC chipset is performed in the frame of a Eureka European co-operation, in partnership with PHILIPS, ST and Thomson Multimedia (MEDEA A101). In this project, the MMIC development work is partly funded by the French Ministry of Industry (STSI). 